\title{
Ginsenoside Rg1 Attenuates Cigarette Smoke-Induced Pulmonary Epithelial-Mesenchymal Transition via Inhibition of the TGF- $\beta 1 /$ Smad Pathway
}

\author{
Sibin Guan, Weiguo Xu, Fengfeng Han, Wen Gu, Lin Song, \\ Wenjing Ye, Qian Liu, and Xuejun Guo
} Department of Respiratory Medicine, Xinhua Hospital, Shanghai Jiao Tong University School of Medicine, 1665 Kongjiang Road,
Shanghai 200092, China

Correspondence should be addressed to Qian Liu; liuqian_1980@hotmail.com and Xuejun Guo; guoxj1964@126.com

Sibin Guan and Weiguo Xu contributed equally to this work.

Received 11 March 2017; Revised 24 May 2017; Accepted 27 June 2017; Published 13 August 2017

Academic Editor: Jeroen T. Buijs

Copyright (c) 2017 Sibin Guan et al. This is an open access article distributed under the Creative Commons Attribution License, which permits unrestricted use, distribution, and reproduction in any medium, provided the original work is properly cited.

\begin{abstract}
Epithelial-mesenchymal transition (EMT) is a process associated with airway remodeling in chronic obstructive pulmonary disease (COPD), which leads to progressive pulmonary destruction. Panax ginseng is a traditional herbal medicine that has been shown to improve pulmonary function and exercise capacity in patients with COPD. Ginsenoside Rg1 is one of the main active components and was shown to inhibit oxidative stress and inflammation. The present study investigated the hypothesis that ginsenoside Rg1 attenuates EMT in COPD rats induced by cigarette smoke (CS) and human bronchial epithelial (HBE) cells exposed to cigarette smoke extract (CSE). Our data showed that CS or CSE exposure increased expression of the mesenchymal marker $\alpha$-smooth muscle actin ( $\alpha$-SMA) and decreased expression of the epithelial marker epithelial cadherin (E-cad) in both lung tissues and HBE cells, which was markedly suppressed by ginsenoside Rg1. Importantly, CS-induced upregulation of TGF- $\beta 1 /$ Smad pathway components, including TGF- $\beta 1$, TGF- $\beta$ R1, phospho-Smad2, and phospho-Smad3, was also inhibited by ginsenoside Rg1. Additionally, ginsenoside Rg1 mimicked the effect of SB525334, a TGF- $\beta$ R1-Smad2/3 inhibitor, on suppression of EMT in CSEinduced HBE cells. Collectively, we concluded that ginsenoside Rgl alleviates CS-induced pulmonary EMT, in both COPD rats and HBE cells, via inhibition of the TGF- $\beta 1 /$ Smad pathway.
\end{abstract}

\section{Introduction}

Chronic obstructive pulmonary disease (COPD), characterized by persistent airflow limitation, remains a leading cause of morbidity and mortality worldwide [1]. It is associated with inflammation and fibrosis, leading to airway remodeling and pulmonary dysfunction $[2,3]$. Current COPD treatments involving $\beta 2$-agonists, anticholinergics, methylxanthines, corticosteroids, phosphodiesterase- 4 inhibitors, rehabilitation, and surgical therapy are effective in reducing symptoms and improving the quality of life of patients with COPD. However, these therapies neither inhibit the progression of COPD towards airway remodeling nor substantively reduce mortality. Therefore, the exploration of novel treatment strategies to prevent airway remodeling is urgently needed.

The mechanisms of airway remodeling in COPD are complex [4]. Recent studies showed that epithelial-mesenchymal transition (EMT), a process in which epithelial cells undergo cytoskeleton reconstruction with loss of cell-cell contacts and acquire a mesenchymal phenotype with excessive extracellular matrix deposition, is involved in the respiratory structural remodeling in COPD [5]. Transitioning epithelial cells acquire enhanced mobility and cross the basal membrane, releasing extracellular matrix proteins that subsequently promote fibrosis and intensify tissue remodeling. Previous 
studies have demonstrated that the transforming growth factor- $\beta 1$ (TGF- $\beta 1$ )/Smad pathway plays a crucial role in triggering EMT [6-8]. The newest study by Mahmood et al. demonstrated that TGF- $\beta 1$ expression was generally higher in COPD subjects throughout the airway wall, while pSmad2/3 expression was associated with smoking, especially in current smoking COPD; in addition, p-Smad was related to airflow obstruction and S100A4 expression [9]. Phosphorylated Smad 2 and Smad3 form a stable complex with Smad4 and then transfer into the nucleus, subsequently modulating the transcription of EMT-associated genes [10]. Besides the Smad cascade, TGF- $\beta 1$ activates EMT via the MEK/ERK, $\mathrm{PI} 3 \mathrm{~K} / \mathrm{AKT}$, and $\mathrm{Wnt} / \beta$-catenin signaling pathways [11-15]. Accordingly, exploring the mechanism of TGF- $\beta 1$-related pathways in COPD could provide attractive therapeutic targets for inhibiting airway remodeling.

Panax ginseng, a traditional herbal medicine, has been widely used in Asian countries for thousands of years. It exhibits multiple pharmacological effects such as antifatigue, anti-inflammation, and antitumor activities, as well as improvement of cognitive function and immunity [1620]. Interestingly, the compound was also shown to enhance pulmonary function and exercise capacity in patients with COPD [21]. Moreover, Sun et al. found that the bioactive substances of ginseng were distributed to various tissues with the highest level in lung [22]. Ginsenoside Rg1 is one of the main active components of Panax ginseng [23], with an oral bioavailability about $18 \%$ [24], and has been reported to attenuate cognitive deterioration, oxidative stress, and inflammation [25-27]. In addition, this compound has been shown to inhibit EMT in hepatic carcinoma and renal tubulointerstitial fibrosis via suppression of TGF- $\beta 1[28,29]$. However, the role of ginsenoside Rg1 in airway remodeling in COPD remains poorly understood.

In the present study, we investigated the effects and the possible mechanism of ginsenoside Rg1 on cigarette smoke- (CS-) induced pulmonary remodeling, with focus on the TGF- $\beta 1 /$ Smad pathway. We found that ginsenoside Rg1 suppressed EMT in vivo following chronic CS exposure and in vitro following cigarette smoke extract (CSE) incubation. These effects were associated with downregulation of TGF- $\beta 1$, TGF- $\beta$ R1, p-Smad2, and p-Smad3. Our findings indicate that ginsenoside $\mathrm{Rgl}$ could act as an attractive therapeutic target for intervention against airway remodeling in COPD.

\section{Methods}

2.1. COPD Rat Model and Experimental Protocols. All animal experiments were approved by the Ethics Committee of Xinhua Hospital affiliated to the Shanghai Jiao Tong University School of Medicine (number XHEC-F-2016-014). Rats were raised in the animal center of Xinhua Hospital affiliated to the Shanghai Jiao Tong University School of Medicine. Cigarettes were purchased from the Shanghai Tobacco Company (Da Qian Men cigarettes: $13 \mathrm{mg}$ tar and $1.3 \mathrm{mg}$ nicotine per cigarette, Shanghai, China). Ginsenoside Rg1, with purity exceeding 98\%, was obtained from Urchem Sinopharm Chemical Reagent Co. Ltd (Lot number 20150511, Shanghai, China).
Forty, male Sprague-Dawley rats $(170-200$ g, aged 8-10 weeks, Shanghai SLAC Laboratory Animal Co., Ltd., Shanghai, China) were randomly divided into five groups ( $n=8$ for each group): normal control, COPD group, COPD with low dose of ginsenoside $\mathrm{Rgl}(5 \mathrm{mg} / \mathrm{kg} \cdot \mathrm{d})$, COPD with medium dose of ginsenoside Rg1 (10 mg/kg.d), and COPD with high dose of ginsenoside $\mathrm{Rg1}(20 \mathrm{mg} / \mathrm{kg} \cdot \mathrm{d})$. COPD rats (COPD group and Rgl groups) were placed in $60 \mathrm{~L}$ Perspex chambers (4 rats/chamber) and exposed to CS generated from Da Qian Men cigarettes. CS was collected by burning 3 cigarettes at one time, 6 times per day, divided into two $1 \mathrm{~h}$ rounds with a $6 \mathrm{~h}$ smoke-free interval, 6 days a week, for 12 weeks. Different doses of ginsenoside Rg1 were intragastrically administered 30 min before CS exposure. Normal group and COPD group were intragastrically given normal saline $(2 \mathrm{ml}$ per animal). Body weight was measured weekly. All rats were sacrificed at the end of week 12. Blood samples were obtained from the abdominal aorta. The right upper lobe lung was fixed in a $4 \%$ neutral formaldehyde solution for pathological and immunohistochemical examinations. The right inferior lobe lung was rapidly reserved in liquid nitrogen and then stored at $-80^{\circ} \mathrm{C}$ for Western blot and real-time PCR analysis.

\subsection{Cell Culture}

2.2.1. CSE Preparation. CSE was prepared by following the method given by Janoff and Carp [30], with modifications. Briefly, a full-length cigarette was combusted through a modified $50 \mathrm{~mL}$ syringe apparatus. The smoke was bubbled through $20 \mathrm{~mL}$ of serum-free RPMI 1640 medium until the unburned butt was less than $5 \mathrm{~mm}$ long. The solution was neutralized with $1 \mathrm{M} \mathrm{NaOH}$ to $\mathrm{pH} 7.4$ and then sterilized through a $0.22 \mu \mathrm{m}$ pore filter. The smoked medium was considered 100\% CSE and diluted with RPMI 1640 medium to the desired concentration, which was used within $30 \mathrm{~min}$ for each experiment.

2.2.2. Cells Culture and Cell Groups. Human bronchial epithelial cells were grown from a BEAS-2B cell line (number CRL-9609; ATCC, Manassas, VA, USA). HBE cells were cultured in Roswell Park Memorial Institute (RPMI) 1640 medium (HyClone, USA) containing 10\% fetal bovine serum (Gibco, Australia) at $37^{\circ} \mathrm{C}$ with $5 \% \mathrm{CO}_{2}$ in humidified air. Cell viability was evaluated by a Cell Counting Kit-8 (CCK-8) assay (Dojindo, Kumamoto, Japan). Ginsenoside Rg1 (20 mg) was dissolved in $20 \mathrm{~mL}$ RPMI 1640 medium sterilized through a $0.22 \mu \mathrm{m}$ pore filter and then diluted to the required concentration. Briefly, $\mathrm{HBE}$ cells were plated onto 96-well plates at a density of $1 \times 10^{4}$ cells per well and then maintained $24-72 \mathrm{~h}$ in $100 \mu \mathrm{L}$ serum-free medium (SFM) for CCK- 8 assay and gene expression detection. When the cells were $60 \%$ confluent, they were treated with different concentrations of $\operatorname{CSE}(0 \%, 5 \%, 10 \%, 15 \%$, and $20 \%)$ or ginsenoside $\operatorname{Rg} 1(5 \mu \mathrm{M}, 10 \mu \mathrm{M}, 20 \mu \mathrm{M}, 40 \mu \mathrm{M}, 80 \mu \mathrm{M}$, and $160 \mu \mathrm{M})$. After $12,24,36,48$, or $72 \mathrm{~h}, 10 \mu \mathrm{L}$ of CCK-8 was added to each well, and the plates were returned to the $37^{\circ} \mathrm{C}$ incubator for $2 \mathrm{~h}$. Absorbance was read at $450 \mathrm{~nm}$ using an ELISA reader (ELx800, BioTek Instruments, USA). Pulmonary EMT was induced with either $10 \%$ CSE or TGF- $\beta 1$ 
(10 ng/ml; PeproTech, USA). HBE cells were divided into nine groups: (1) normal, (2) CSE, (3) Rg1 (40 $\mu \mathrm{M})$, (4) SB525334 ( $3 \mu \mathrm{M}$; Selleck Chem., USA), (5) CSE + Rg1 (40 $\mu \mathrm{M})$, (6) $\mathrm{CSE}+\mathrm{SB} 525334(3 \mu \mathrm{M}),(7) \mathrm{CSE}+\operatorname{Rg} 1(40 \mu \mathrm{M})+\mathrm{SB} 525334$ $(3 \mu \mathrm{M}),(8) \mathrm{TGF}-\beta 1$, and (9) TGF- $\beta 1+\operatorname{Rg} 1(40 \mu \mathrm{M})$. After $48 \mathrm{~h}$ of incubation, cells were observed using a light microscope, and images were obtained with the Leica Application Suite program. Cells were collected and subjected to Western blot analysis.

2.3. Measurement of TGF- $\beta 1$ in Serum. Concentration of TGF- $\beta 1$ in serum was measured by ELISA kits (eBioscience, USA) according to the manufacturer's instructions. The optical density of each sample was read at $45 \mathrm{~nm}$.

2.4. Lung Histopathology. The lung tissues were embedded in paraffin and cut into $4 \mu \mathrm{m}$ thick sections, which were then rehydrated and stained with haematoxylin and eosin to evaluate pulmonary architecture. To access the degree of fibrosis, Masson trichrome staining was performed. Histological changes in each sample were examined under the light microscope. The extent of fibrosis was determined by calculating the blue area normalized to whole pulmonary area.

2.5. Real-Time PCR Analysis. Total RNA was extracted from the lung tissue and reverse-transcribed to cDNA following the method as previously described [31]. Primer sequences (synthesized by Sangon Biotech, China) for target genes (Ecad, $\alpha$-SMA, and TGF- $\beta 1$ ) were as follows: Fwd $5^{\prime}$-TCTCTTGTCCCTTCCACAGC- $3^{\prime}$ and Rev $5^{\prime}$-CTCCAGACCCACACCAAAGT- $3^{\prime}$ for E-cad; Fwd $5^{\prime}$-TTCGTGACTACTGCTGAGCG- $3^{\prime}$ and Rev $5^{\prime}$-CTGTCAGCAATGCCTGGGTA-3' for $\alpha$-SMA; Fwd $5^{\prime}$-ATTCCTGGCGTTACCTTGG- $3^{\prime}$ and Rev $5^{\prime}$-AGCCCTGTATTCCGTCTCCT- $3^{\prime}$ for TGF- $\beta 1$. For all lung specimens, glyceraldehyde 3-phosphate dehydrogenase (GAPDH) mRNA (Fwd 5' -GGCACAGTCAAGGCTGAGAATG-3' and Rev 5'-ATGGTGGTGAAGACGCCAGTA-3') was amplified as an internal control. To improve the accuracy of real-time PCR results, amplifications were performed in triplicate for each RNA sample.

2.6. Western Blot Analysis. Lung tissues and $\mathrm{HBE}$ were lysed in RIPA buffer (Beyotime Ins., China) containing $10 \mu \mathrm{L} / \mathrm{mL}$ of phenylmethylsulfonyl fluoride (PMSF) and $10 \mu \mathrm{L} / \mathrm{mL}$ of phosphatase inhibitor. Protein samples were separated via $6-10 \%$ sodium dodecyl sulfate polyacrylamide gel electrophoresis (SDS-PAGE) and were transferred to polyvinylidene fluoride membranes (Millipore, Billerica, USA). Membranes were blocked in 5\% nonfat milk for $2 \mathrm{~h}$ at room temperature and then incubated with primary antibodies against E-cad, $\alpha$-SMA, vimentin, TGF- $\beta 1$, TGF$\beta R 1$, phospho-Smad2 ( $\mathrm{p}-\mathrm{Smad} 2$ ), total Smad2, phosphoSmad3 (p-Smad3), or total Smad3 (Abcam, USA) overnight at $4^{\circ} \mathrm{C}$. GAPDH (Sigma-Aldrich, USA) was used as the internal control. All primary antibodies could react with both rat and human. After treatment with horseradish peroxidase (HRP) secondary anti-mouse or anti-rabbit antibodies, imaging was performed using enhanced chemiluminescence fluid (Millipore Corporation, USA).

2.7. Statistical Analysis. Data were expressed as mean \pm standard error of the mean. All graphs and statistical analyses were performed using GraphPad Prism 5 software (GraphPad Software, Inc., La Jolla, CA, USA). One-way ANOVA and Tukey's post hoc test were performed to determine differences among groups. $P<0.05$ and $P<0.01$ were considered statistically significant.

\section{Results}

3.1. Ginsenoside Rg1 Alleviated Cigarette Smoke-Induced Inflammation and Fibrosis. COPD is a progressive pulmonary disease that is primarily caused by CS. CS exposure induces chronic inflammation, emphysema, and lung fibrosis, leading to airway remodeling. In comparison to normal group, obvious adhesion, lodging and shedding of cilia, and marked alveolar ectasia were observed in COPD group. These changes were reduced by addition of ginsenoside Rg1 (Figure 1(a)). Masson trichrome staining revealed that, compared with normal group, CS exposure significantly increased pulmonary interstitial fibrosis, which was attenuated with ginsenoside Rg1 treatment (Figure 1(b)). A quantitative analysis produced consistent results (Figure $1(\mathrm{c})$ ). The interstitial fibrosis caused by CS was significantly decreased in ginsenoside Rg1 treated groups $(5 \mathrm{mg} / \mathrm{kg}, 10 \mathrm{mg} / \mathrm{kg}$, and $20 \mathrm{mg} / \mathrm{kg}$ ) compared with COPD group $(15.13 \% \pm 1.55 \%$, $11.80 \% \pm 1.39 \%$, and $8.81 \% \pm 1.69 \%$ versus $19.64 \pm 2.16 \%$, $P<0.01)$. These data indicated that ginsenoside Rg1 treatment inhibited CS-induced emphysema and airway fibrosis.

3.2. Ginsenoside Rg1 Attenuated CS-Induced EMT in Rat Lung Tissue. EMT is characterized by loss of the epithelial marker E-cad and acquisition of the mesenchymal markers $\alpha$ SMA and vimentin. As shown in Figures 2(a)-2(d), Western blots indicated that $\alpha$-SMA and vimentin expressions were markedly increased, whereas E-cad expression decreased slightly in COPD group compared with that in normal group. Ginsenoside Rg1 treatment at 5, 10, and $20 \mathrm{mg} / \mathrm{kg}$ markedly decreased $\alpha$-SMA and vimentin expressions and restored Ecad level in a dose-dependent manner $(P<0.05$ versus COPD group). Real-time PCR showed similar effects of ginsenoside Rg1 on E-cad and $\alpha$-SMA expressions to those observed in Western blots (Figures 2(e) and 2(f)).

3.3. Ginsenoside Rg1 Decreased TGF- $\beta 1$ Production in Serum and Suppressed TGF- $\beta 1 /$ Smad Signaling Expression in Lung Tissue of COPD Rats. TGF- $\beta$-particularly the TGF- $\beta 1$ isoform-plays a key role in driving EMT. Previous studies have confirmed that TGF- $\beta 1 /$ Smad pathway takes part in EMT. To investigate the potential mechanism by which ginsenoside Rg1 inhibited EMT in the COPD model, we investigated the effects of ginsenoside Rg1 on TGF- $\beta 1$ content in the serum and the mRNA and protein expression of TGF- $\beta 1$ in lung tissue of COPD rats. CS exposure increased serum TGF- $\beta 1$ levels (Figure $3(\mathrm{a})$ ) and enhanced TGF- $\beta 1$ 


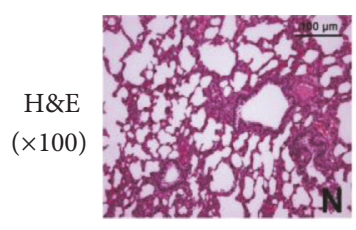

Normal

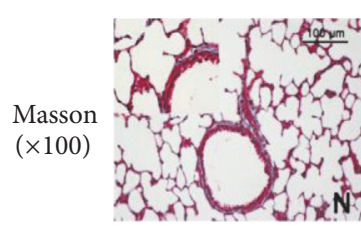

Normal

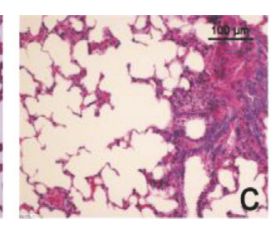

COPD

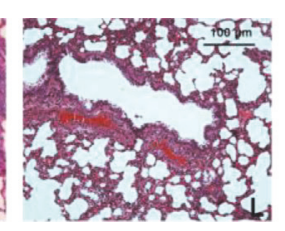

Rg1-5 mg

(a)

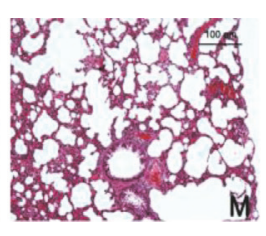

Rg1-10 mg

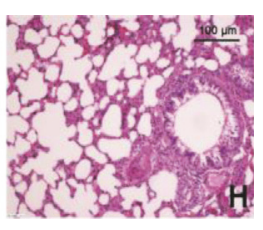

Rg1-20 mg

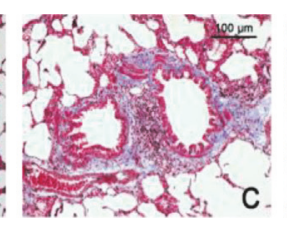

COPD

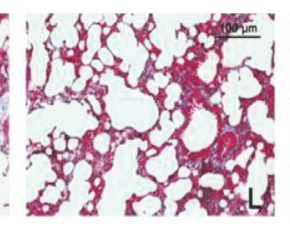

Rg1-5 mg

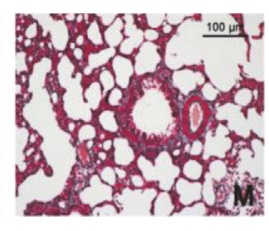

Rg1-10 mg

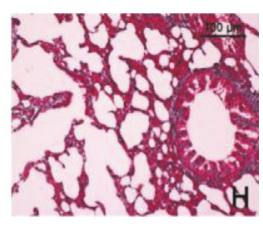

Rg1-20 mg

(b)

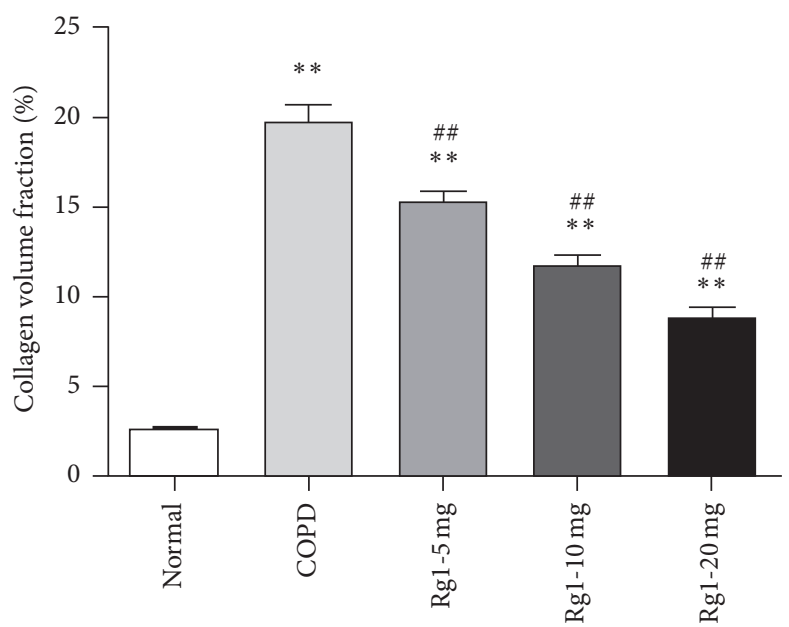

(c)

Figure 1: Ginsenoside Rg1 alleviated CS-induced emphysema and fibrosis. (a) Lung histology was analyzed via H\&E staining ( $\times 100$ magnification). (b) Lung histology was analyzed via Masson trichrome staining ( $\times 100$ magnification). (c) Quantitative analysis of collagen in lung tissue was carried out using Image-Pro Plus 6.0 software. The percentage of the positive staining area of the airway was counted from 6 randomly selected fields per section. Mean optical densities were measured. All data are shown as the mean \pm SD; $n=6$ per group. Statistical significance was assessed by one-way ANOVA and Tukey's post hoc test. ${ }^{* *} P<0.01$ versus normal group; ${ }^{\# \#} P<0.01$ versus COPD group.

mRNA and protein expression compared with normal group. However, ginsenoside $\mathrm{Rgl}$ at 5,10 , and $20 \mathrm{mg} / \mathrm{kg}$ reduced the TGF- $\beta 1$ level in serum $(66.98 \pm 6.57,59.53 \pm 2.82$, and 47.55 \pm 4.32 versus $81.03 \pm 8.41, P<0.05)$ and downregulated the mRNA (3.55-, 2.39-, and 1.61-fold versus 5.54-fold, $P<$ $0.05)$ and protein expression of TGF- $\beta 1(0.82 \pm 0.09,0.63$ \pm 0.13 , and $0.49 \pm 0.07$ versus $1.16 \pm 0.20, P<0.05)$ compared with COPD group (Figures $3(\mathrm{~b})$ and $3(\mathrm{c})$ ). We also evaluated different components of TGF- $\beta 1 /$ Smad signaling in the lung tissues by Western blot. As shown in Figure 3(c), the protein levels of TGF- $\beta$ R1, p-Smad2, and p-Smad 3 in COPD group were significantly higher than those of normal group, while ginsenoside Rgl treatment downregulated expressions of TGF- $\beta$ R1, p-Smad2, and p-Smad3, especially at a high dose. These results suggested that ginsenoside Rg1 attenuated the EMT at least in part through the inhibition of TGF- $\beta 1$ production, which could be associated with the inhibition of TGF- $\beta 1 /$ Smad signaling pathway.

3.4. Cell Viability. A CCK- 8 assay was used to determine changes in cell viability after $\mathrm{HBE}$ cells were incubated with CSE $(0 \%, 5 \%, 10 \%, 15 \%$, and $20 \%)$ or ginsenoside $\operatorname{Rg} 1(5 \mu \mathrm{M}$, $10 \mu \mathrm{M}, 20 \mu \mathrm{M}, 40 \mu \mathrm{M}, 80 \mu \mathrm{M}$, and $160 \mu \mathrm{M})$ for $72 \mathrm{~h}$. As shown in Figure 4(a), HBE cells being exposed to CSE exhibited a dose-dependent decrease in cell viability. After $60 \mathrm{~h}$, all CSE groups displayed striking decreases (up to $70 \%$ ) in the $\mathrm{HBE}$ cell viability. CSE incubation greater than $48 \mathrm{~h}$ resulted in significant toxicity ( $>50 \%$ viability decline) to $\mathrm{HBE}$ cells. Next, we estimated the effect of ginsenoside Rgl on HBE cell viability. When cells were incubated with $80 \mu \mathrm{M}$ and $160 \mu \mathrm{M}$ ginsenoside Rgl for $72 \mathrm{~h}$, cell viability decreased by $13.2 \%$ and $20.7 \%$, respectively (Figure $4(\mathrm{~b})$ ). Treatment with 5-40 $\mu \mathrm{M}$ 


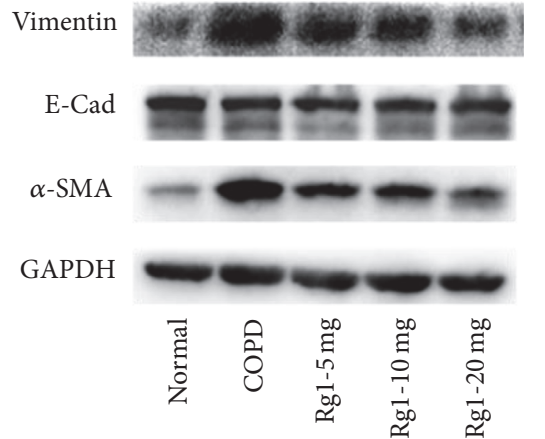

(a)

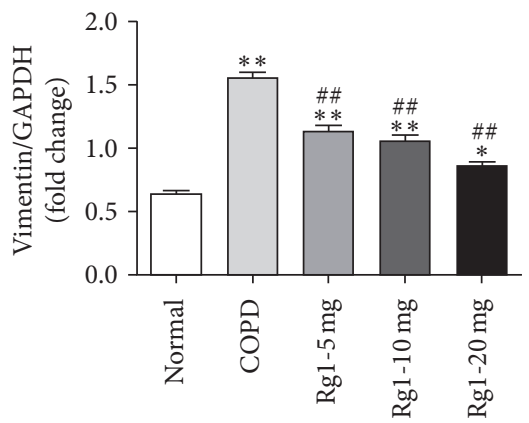

(d)

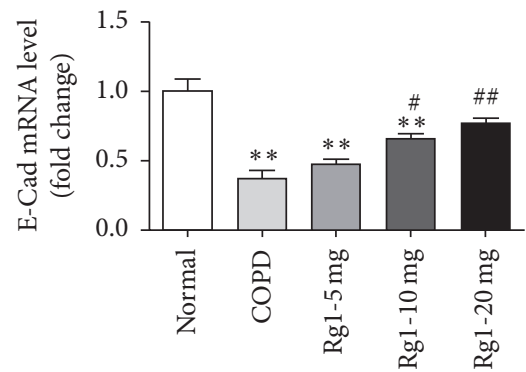

(b)

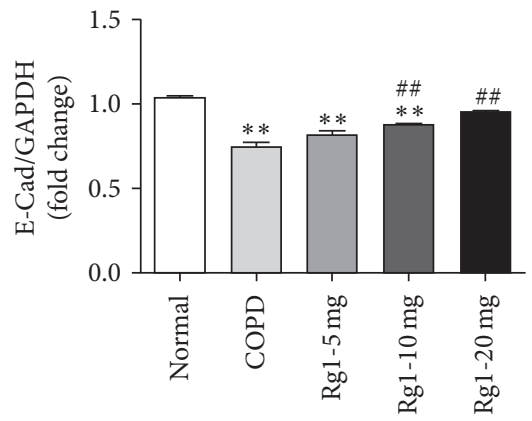

(e)

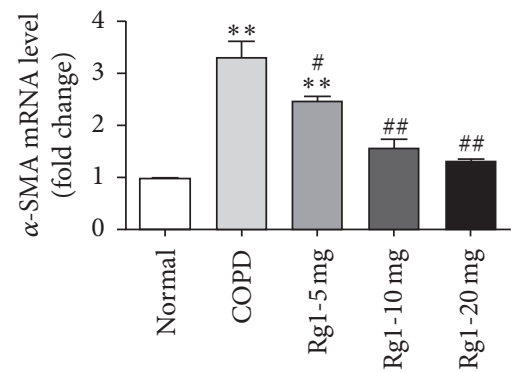

(c)

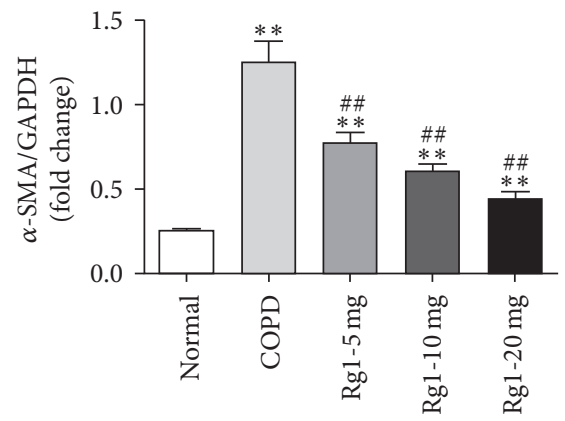

(f)

FIGURE 2: Ginsenoside Rg1 alleviated CS-induced EMT in lung tissues. (a) The expressions of E-cad, $\alpha$-SMA, and vimentin were measured by Western blots ((a), (b), (c), and (d)). The expressions of E-cad and $\alpha$-SMA were measured by real-time PCR ((e) and (f)). All data were shown as the mean $\pm \mathrm{SD} ; n=6$. Statistical significance was assessed by one-way ANOVA and Tukey's post hoc test. ${ }^{*} P<0.05$ and ${ }^{* *} P<0.01$ versus normal group; ${ }^{\#} P<0.05$ and ${ }^{\# \#} P<0.01$ versus COPD group.

ginsenoside Rg1 showed a minor effect on $\mathrm{HBE}$ cell viability. Therefore, HBE cells were exposed to $10 \%$ CSE and/or $40 \mu \mathrm{M}$ ginsenoside $\mathrm{Rg} 1$ for $48 \mathrm{~h}$ in the following experiments.

3.5. Ginsenoside Rg1 Protected HBE Cells against EMT Induced by CSE or TGF- $\beta 1$. To investigate the role of ginsenoside $\mathrm{Rg} 1$ in regulating $\mathrm{BE}$ cells transdifferentiation, BE cells were stimulated with $10 \%$ CSE or TGF- $\beta 1(10 \mathrm{ng} / \mathrm{mL})$ in the presence or absence of ginsenoside $\operatorname{Rg} 1(40 \mu \mathrm{M})$ for $48 \mathrm{~h}$. First, we evaluated the morphological changes of HBE cells by light microscopy $(40 \mathrm{x})$. The results showed that BE cells changed from cobblestone appearance to long spindle shape in response to CSE exposure. Treatment with ginsenoside Rg1 alleviated the morphological changes (Figures 4(c)-4(f)). Next, HBE cells were collected and subjected to Western blots to detect the protein level of $\alpha$-SMA and E-cad. In accordance with the in vivo results, our in vitro data indicated that CSE stimulation significantly increased protein expression of $\alpha$-SMA and slightly decreased E-cad protein expression in $\mathrm{HBE}$ cells compared with those of the normal group. In the CSE + Rgl group, the protein level of $\alpha$-SMA was markedly decreased $(0.85 \pm 0.11$ versus $1.34 \pm 0.08, P<0.01$ versus COPD group), whereas E-cad protein level was increased $(0.71 \pm 0.09$ versus $0.49 \pm 0.08, P<0.05$ versus COPD group, Figures 4(g)-4(i)). Additionally, compared with TGF- $\beta 1$ alone, ginsenoside $\mathrm{Rg} 1$ administration significantly reduced expression of $\alpha$-SMA $(0.70 \pm 0.07$ versus $1.07 \pm 0.15, P<0.01$ versus COPD group) and upregulated the E-cad level (0.74 \pm 0.06 versus $0.55 \pm 0.07, P<0.05$ versus COPD group). We concluded that ginsenoside Rg1 suppressed the EMT induced by CSE and exhibited similar protective effect on HBE cells treated with TGF- $\beta 1$ (Figures $4(\mathrm{j})-4(\mathrm{l})$ ).

3.6. Smad2 and Smad3 Are Activated in CS-Stimulated HBE Cells and Are Inhibited by Ginsenoside Rg1 Treatment. Previous study demonstrated that Panax ginseng could inhibit Smad2 and Smad3 activation [32]. Because the classic signal that regulated EMT is mediated by TGF- $\beta 1 /$ Smad pathway, we tested whether Smad2 and Smad3 were activated in HBE cells in response to CSE stimulation. HBE cells were treated with 10\% CSE for different intervals $(0,15,30$, and $60 \mathrm{~min})$ and then the cell protein was extracted for Western blot analysis of total Smad2, p-Smad2, total Smad3, and p-Smad3. We found that the levels of p-Smad2 and p-Smad3, which were low before CSE stimulation, significantly increased after CSE stimulation and the phosphorylations were the highest at $30 \mathrm{~min}$ in response to CSE treatment in HBE cells. By contrast, with ginsenoside Rg1 administration, there was a marked decrease in p-Smad2 and p-Smad3 (Figure 5). Our data suggested that CSE stimulation dramatically enhanced the activation-associated phosphorylation of Smad2 and Smad3. Additionally, ginsenoside Rgl treatment inhibited the activation of Smad2 and Smad3. 


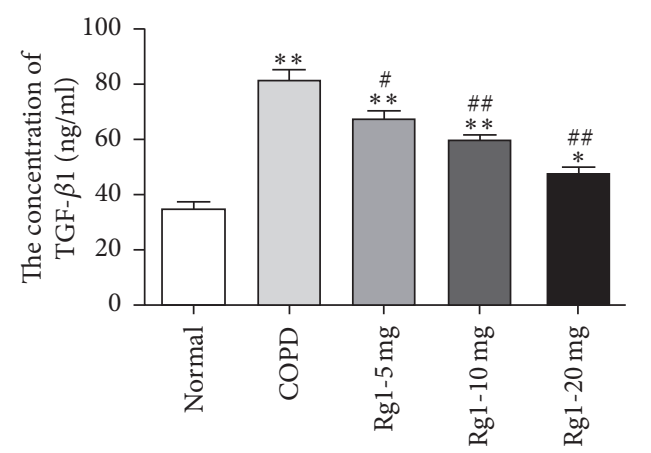

(a)

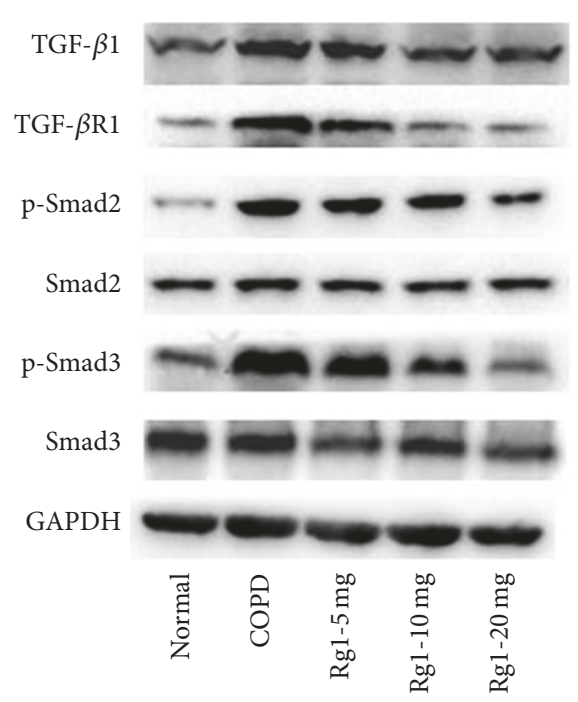

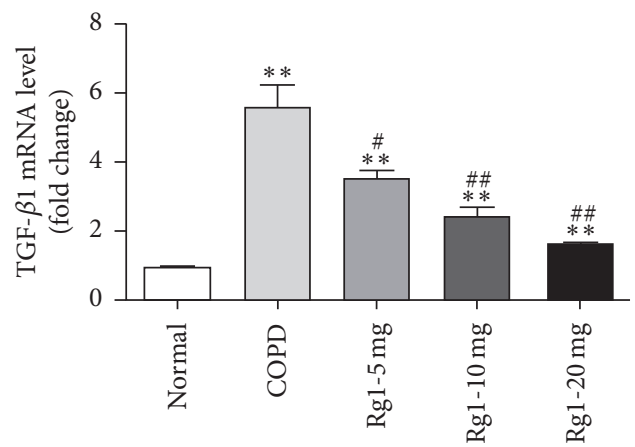

(b)
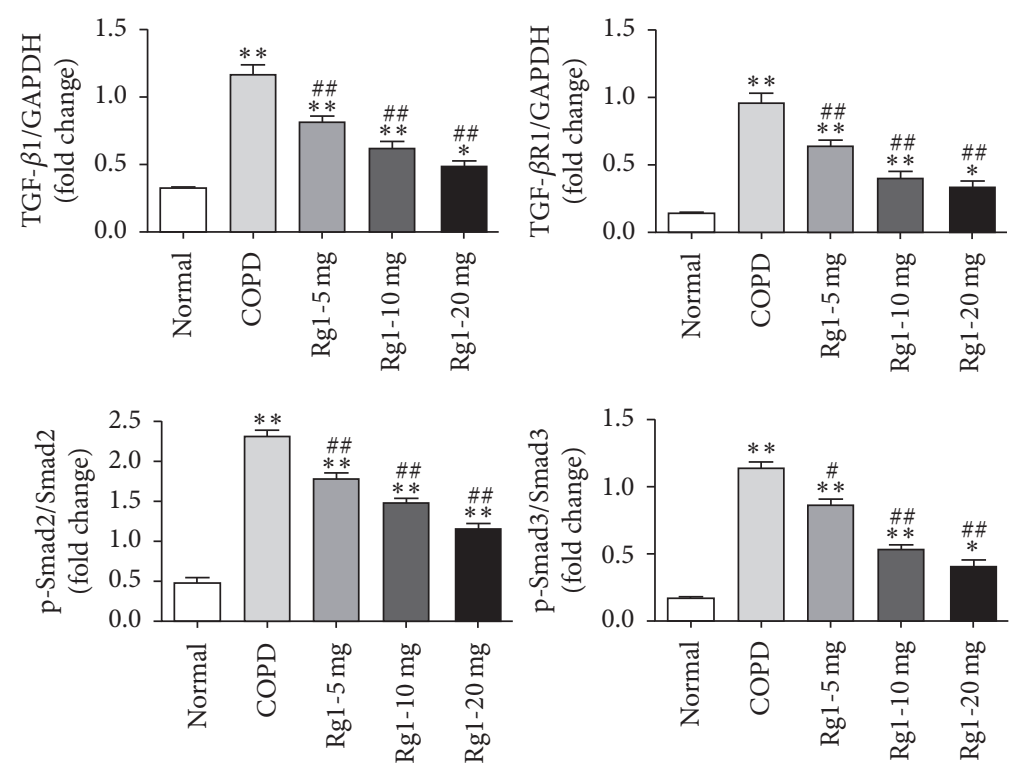

(c)

FIGURE 3: Ginsenoside Rg1 decreased TGF- $\beta 1$ production in serum and suppressed TGF- $\beta 1 /$ Smad signaling expression in lung tissue of COPD rats. (a) TGF- $\beta 1$ expression in the serum was determined by ELISA. (b) The mRNA level of TGF- $\beta 1$ in lung tissue was determined by real-time PCR. (c) Protein levels of TGF- $\beta 1$, TGF- $\beta$ R1, p-Smad2, total Smad2, p-Smad3, and total Smad3 were determined by Western blot. Data were expressed as the mean $\pm \mathrm{SD} ; n=6$. Statistical significance was assessed by one-way ANOVA and Tukey's post hoc test. ${ }^{*} P<0.05$ and ${ }^{* *} P<0.01$ versus normal group; ${ }^{\#} P<0.05$ and ${ }^{\# \#} P<0.01$ versus COPD group.

3.7. Inhibition of Smad2 and Smad3 Enhanced the Ginsenoside Rg1-Mediated Effect on CS-Induced EMT in HBE Cells. To further explore the effects of ginsenoside Rg1 on the regulation TGF- $\beta 1 / \mathrm{Smad}$ pathway, we investigated the effect of ginsenoside Rg1 on EMT using SB525334, a specific inhibitor of activin receptor-like kinase 5 (ALK5), which could block the activation of $\operatorname{Smad} 2 / 3$. We first noted no marked difference in E-cad or $\alpha$-SMA protein levels among normal HBE cells and HBE cells treated with ginsenoside Rg1 or SB525334. As expected, pretreatment with SB525334 prior to CSE administration markedly suppressed $\alpha$-SMA and increased E-cad expression at protein level, demonstrating that the TGF- $\beta 1 /$ Smad pathway is responsible for CSEinduced EMT in HBE cells. There was no statistical difference between CSE + SB525334 group and CSE + Rg1 group. Furthermore, the reduction of $\alpha$-SMA and the restoration of E-cad regulated by ginsenoside Rg1 were enhanced by adding SB525334. These data indicated that ginsenoside Rg1 suppressed pulmonary EMT at least partly through TGF$\beta 1 /$ Smad inhibition (Figure 6).

\section{Discussion}

Airway remodeling and emphysema trigger progressive impairment of pulmonary function [33]. Emerging evidence suggests that EMT may lead to the genesis of airway remodeling, indicating a close association between EMT and pulmonary function [4,5]. Gross et al. and Shergis et al. reported the protective effects of ginseng and ginsenosides on lung function and quality of life in COPD [21,34]. To better understand the effect of ginsenoside Rg1 on airway remodeling, we treated CS-induced COPD rats with ginsenoside Rg1. The present study indicated that ginsenoside Rg1 decreased $\alpha$-SMA expression, restored the decreased expression of Ecad, a specific epithelial marker in lung tissue, and decreased 


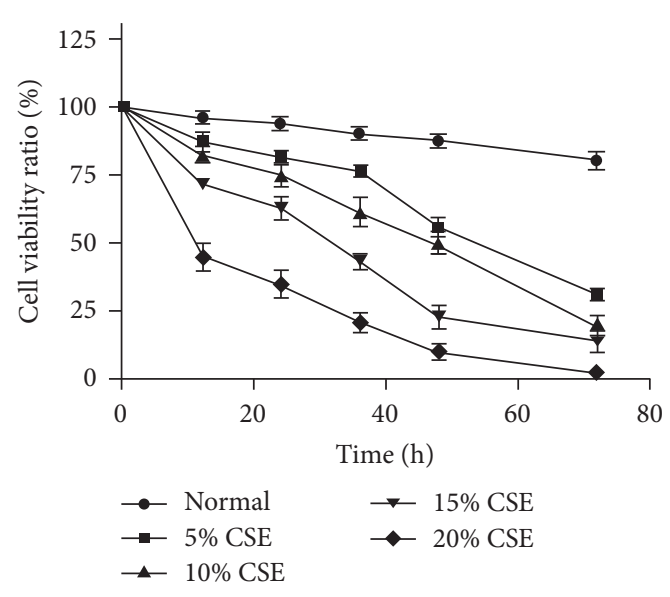

(a)

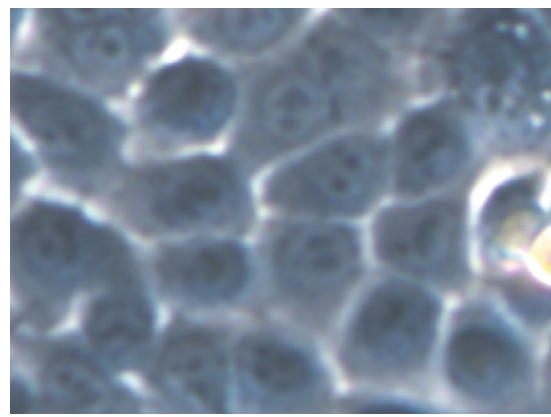

Sham

(c)

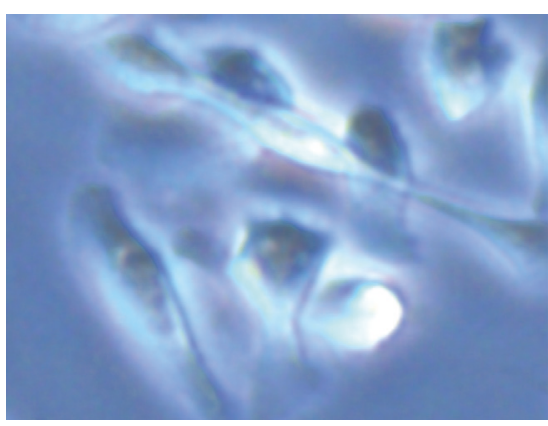

CSE

(e)

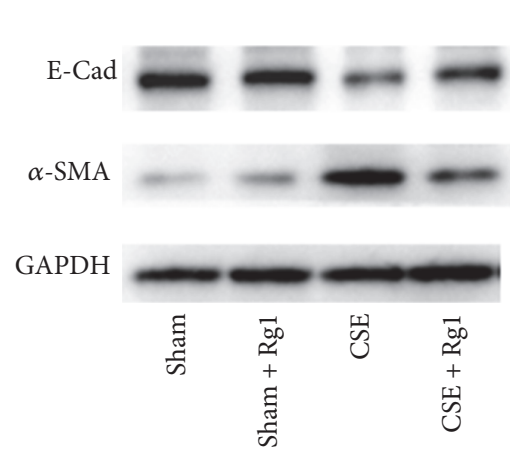

(g)

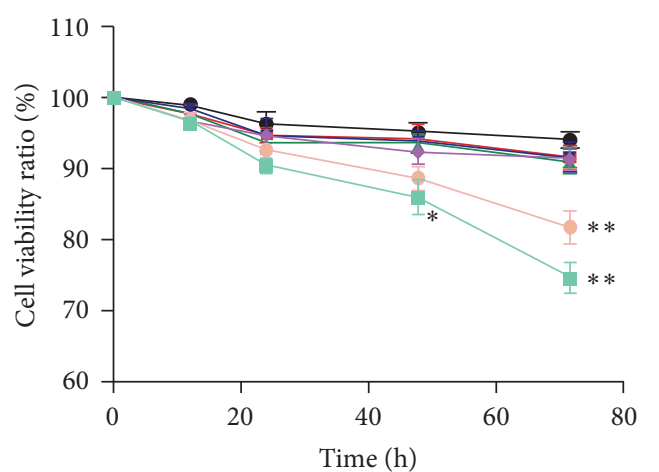

$$
\begin{aligned}
& \text { - Sham } \quad \bullet 40 \mu \mathrm{M} \mathrm{Rg} 1 \\
& \rightarrow 5 \mu \mathrm{M} \mathrm{Rg} 1 \quad-80 \mu \mathrm{M} \mathrm{Rg} 1 \\
& \longrightarrow 10 \mu \mathrm{MRg} 1 \rightarrow 160 \mu \mathrm{M} \mathrm{Rg} 1 \\
& \rightarrow 20 \mu \mathrm{M} \text { Rg1 }
\end{aligned}
$$

(b)

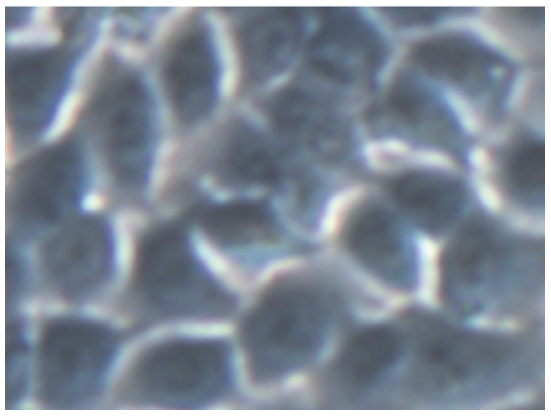

Sham + Rg1

(d)

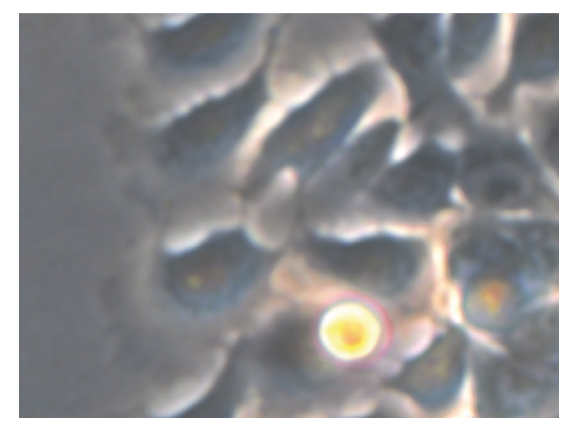

$\mathrm{CSE}+\operatorname{Rg} 1$

(f)

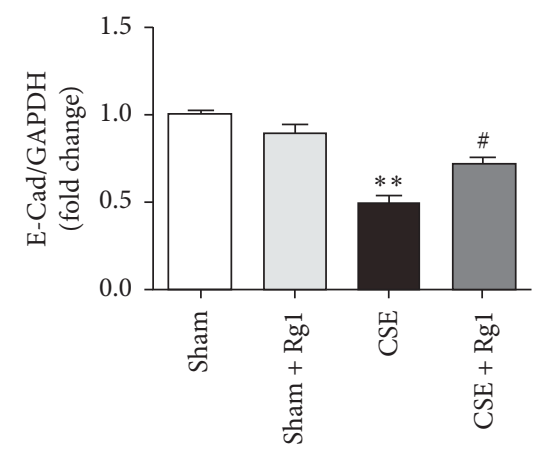

(h)

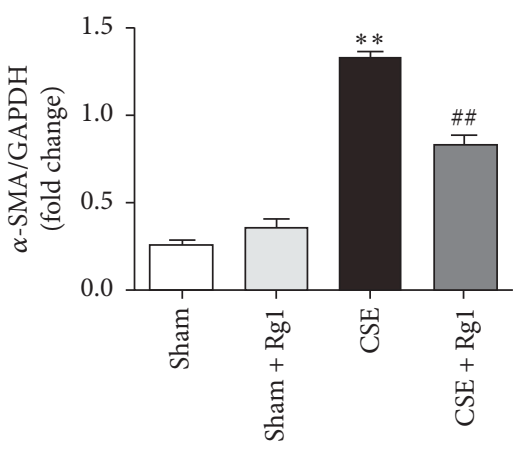

(i)

FIGURE 4: Continued. 


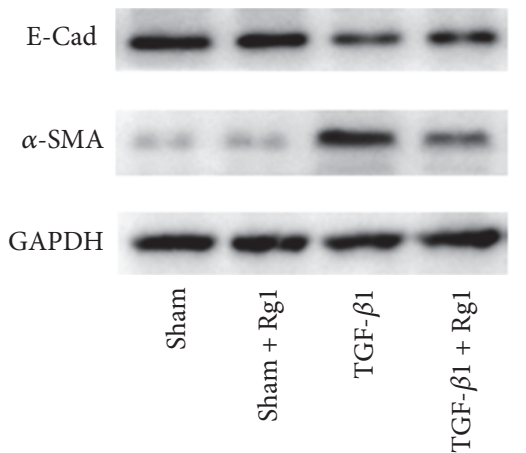

(j)

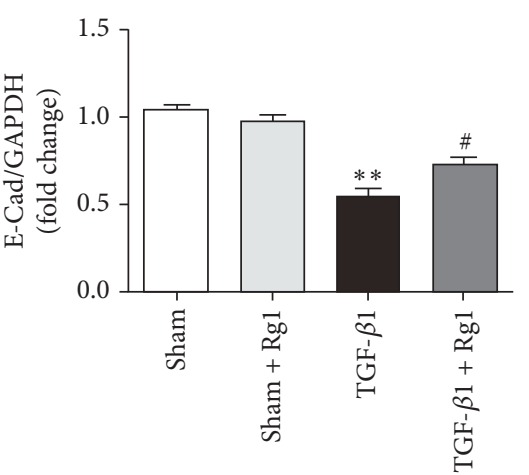

$(\mathrm{k})$

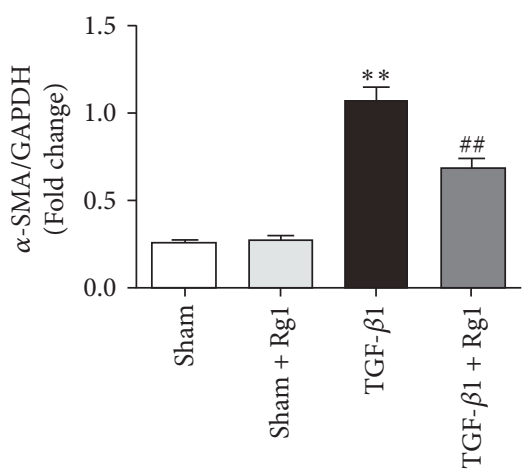

(1)

Figure 4: Ginsenoside Rg1 protected HBE cells against CSE or TGF- $\beta 1$-induced EMT. ((a) and (b)) Effects of CSE and ginsenoside Rg1 on the cell viability were measured using a CCK- 8 assay. ((c), (d), (e), and (f)) Light microscopy of HBE cells $(\times 100$ magnification). ((g), (h), and (i)) HBE cells were induced with $10 \% \mathrm{CSE}$ and treated with ginsenoside Rg1 $(40 \mu \mathrm{M})$ for $48 \mathrm{~h}$. Protein expressions of E-cad and $\alpha$-SMA were determined by Western blot. ((j), (k), and (1)) HBE cells were induced with TGF- $\beta 1(10 \mathrm{ng} / \mathrm{ml})$ and treated with ginsenoside Rg1 (40 $\mu \mathrm{M})$ for $48 \mathrm{~h}$. Protein expressions of E-cad and $\alpha$-SMA were determined by Western blot. Data were expressed as the mean $\pm \mathrm{SD} ; n=5$. Statistical significance was assessed by one-way ANOVA and Tukey's post hoc test. ${ }^{*} P<0.05$ and ${ }^{* *} P<0.01$ versus corresponding untreated controls; ${ }^{\#} P<0.05$ and ${ }^{\# \#} P<0.01$ versus corresponding ginsenoside Rg1 (-) group.

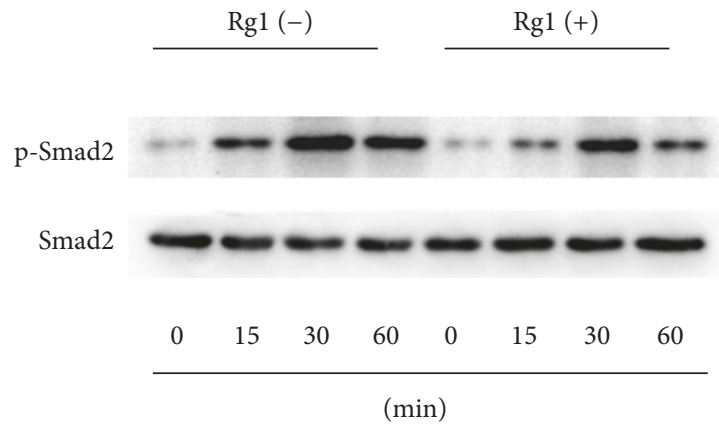

(a)

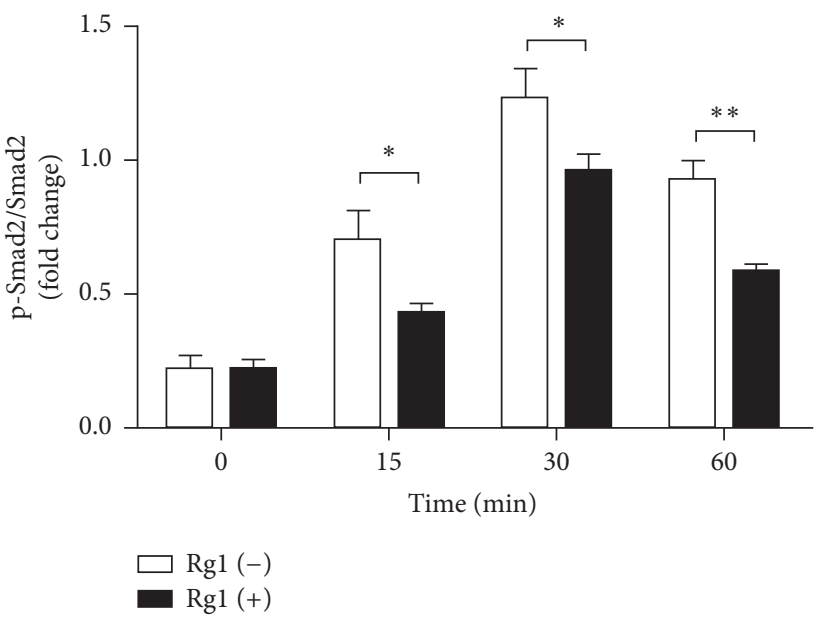

(c)

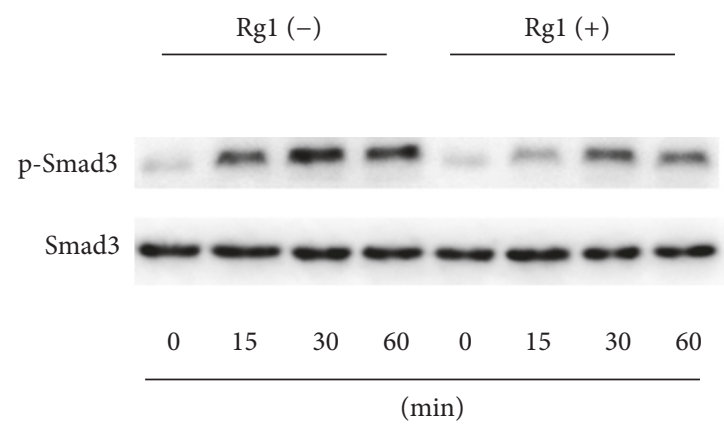

(b)

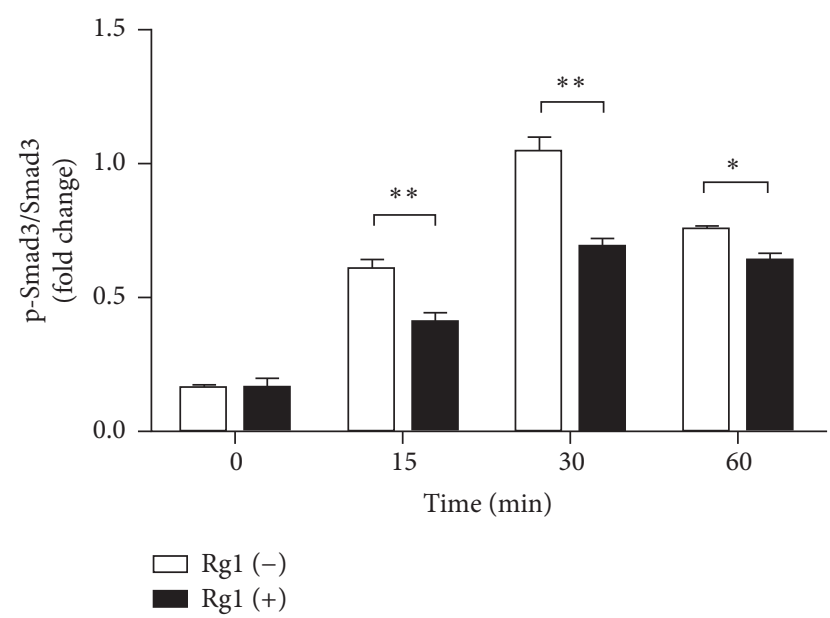

(d)

FIGURE 5: Ginsenoside Rg1 suppressed CS-induced phosphorylation of Smad2 and Smad3 in HBE cells. (a) HBE cells were treated with 10\% CSE for 0-60 min; Smad2 was phosphorylated by CSE with maximal phosphorylation at 30 min; the phosphorylation was significantly suppressed by ginsenoside $\operatorname{Rg} 1(40 \mu \mathrm{M})$ treatment. (b) Smad3 was phosphorylated in response to CSE stimulation and peaked at 30 min; the phosphorylation could be abated by ginsenoside $\operatorname{Rg} 1(40 \mu \mathrm{M})$ treatment. Data were expressed as the mean \pm SD; $n=3$ per group. Statistical significance was assessed by two-way ANOVA and Bonferroni's posttest. ${ }^{*} P<0.05$ and ${ }^{* *} P<0.01$. 


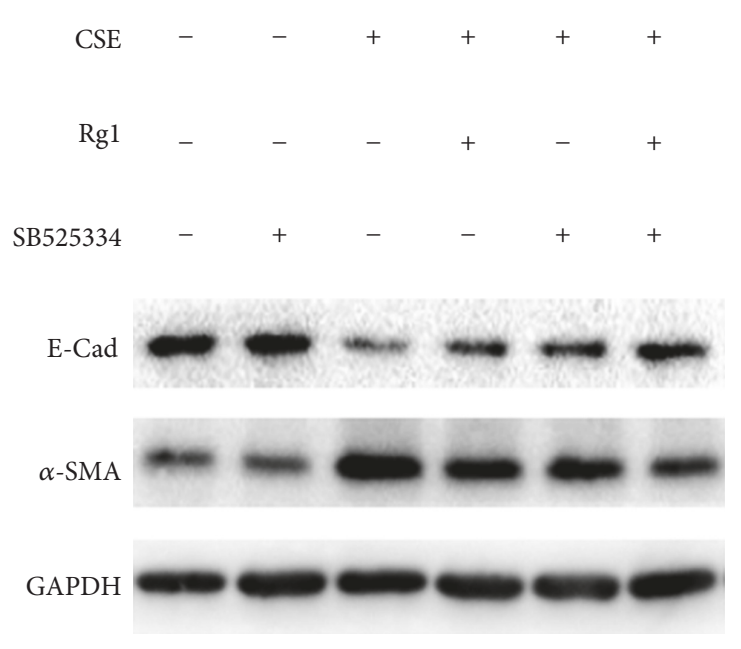

(a)

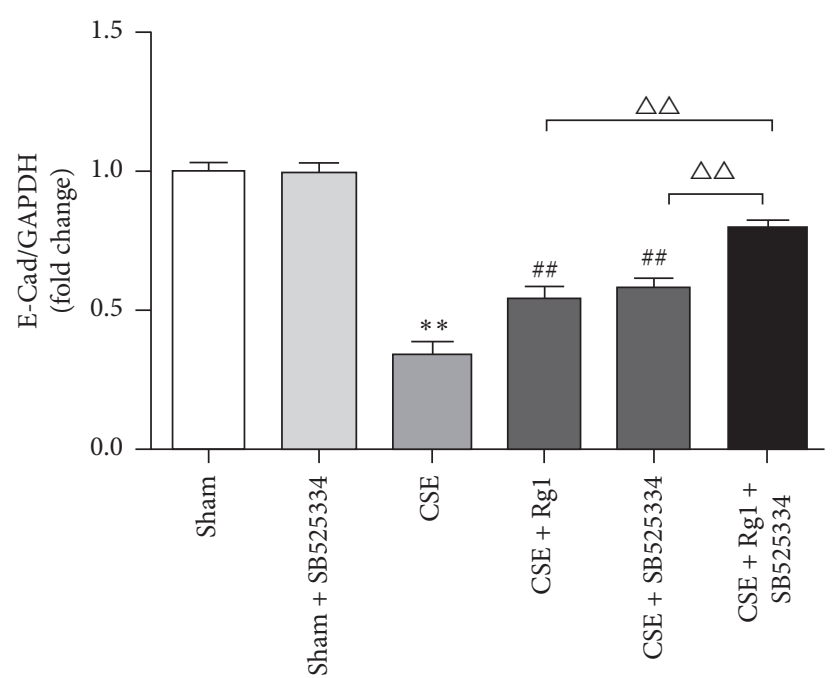

(b)

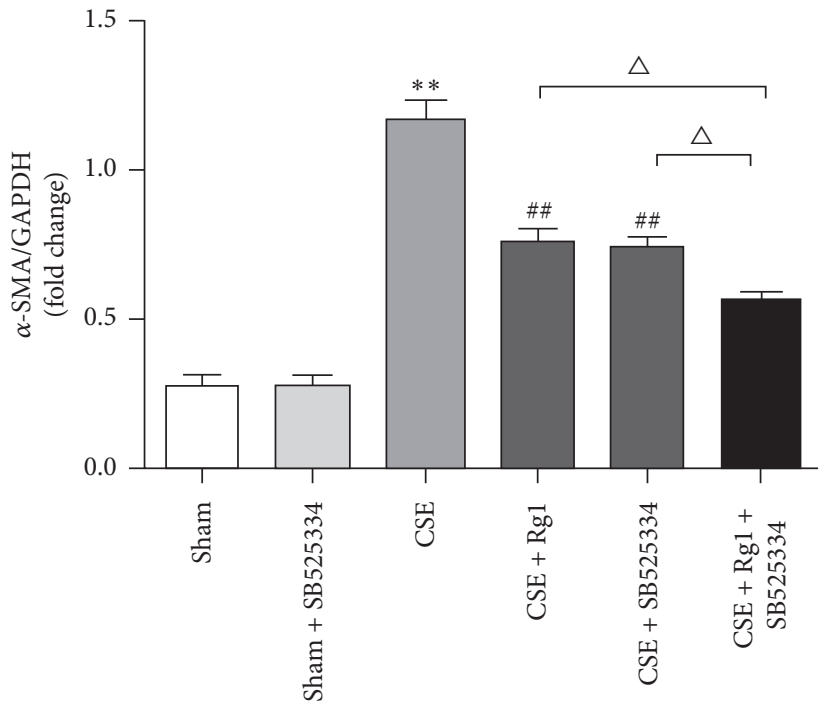

(c)

FIGURE 6: Inhibition of Smad2 and Smad3 abrogated the ginsenoside Rg1-mediated effect on CSE-induced EMT in HBE cells. HBE cells were pretreated with or without SB525334 $(3 \mu \mathrm{M})$ for $60 \mathrm{~min}$ and then stimulated with 10\% CSE and cultured with or without ginsenoside Rg1 $(40 \mu \mathrm{M})$ for $48 \mathrm{~h}$. Protein expressions of E-cad and $\alpha$-SMA were determined by Western blot. Data were expressed as the mean $\pm \mathrm{SD} ; n=6$. Statistical significance was assessed by one-way ANOVA and Tukey's post hoc test. ${ }^{* *} P<0.01$ versus Sham group; ${ }^{\# \#} P<0.01$ versus CSE group. ${ }^{\triangle} P<0.05$ and ${ }^{\triangle \triangle} P<0.01$ versus CSE + Rg1 + SB525334 group.

pulmonary fibrosis in COPD rats. These results indicated the inhibitory effect of ginsenoside Rgl on EMT. Similar effects of ginsenoside Rgl were observed in HBE cells treated with CSE or TGF- $\beta 1$. Importantly, our findings revealed that these effects were mediated in part through suppression of TGF$\beta 1 /$ Smad signaling pathway, suggesting that ginsenoside Rg1 could be a therapeutic target for airway remodeling in COPD.

EMT is a vital process during wound healing and tissue repair but it can also induce complete tissue fibrosis if the damage or inflammation persists, which has been demonstrated in the liver, kidney, and bowel [35-37]. Recent studies have shown that EMT plays a homeostatic role in the airway response to injury and stress and participates in the pathogenesis of COPD, asthma, and idiopathic pulmonary fibrosis [38-40]. In agreement with the work by Mahmood et al. [38], we observed by immunohistochemical staining that rats exposed to CS for 12 weeks exhibited upregulation of $\alpha$-SMA and downregulation of E-cad in lung tissue; moreover, these findings were validated by quantifying RNA and protein levels of these molecules. The above variations in vivo could be countered in a dose-dependent manner by ginsenoside Rg1 administration. At the cellular level, Western blot analysis revealed enhanced $\alpha$-SMA expression and decreased E-cad expression in HBE cells treated with CSE or TGF- $\beta 1$, and these effects could be reversed by ginsenoside Rgl. The histological analysis of the hematoxylin and eosin and Masson trichrome-stained sections of COPD rat lung tissue indicated that ginsenoside Rg1 treatment decreased 
emphysema and interstitial fibrosis induced by CS. Taken together, these findings demonstrate that ginsenoside Rg1 treatment suppresses both CS-induced pulmonary EMT and TGF- $\beta 1$-induced EMT in HBE cells.

TGF- $\beta 1$ has been implicated as a "master switch" in the induction of EMT. In vitro studies demonstrated that exposure to TGF- $\beta 1$ induces different pulmonary epithelial cells to acquire a mesenchymal phenotype [41-43]. Activation of the TGF- $\beta 1 /$ Smad signaling pathway plays a critical role in the pathogenesis of EMT. Kasai et al. reported that the knockdown of Smad2 with siRNA restored the decreased expression of E-cad in A549 cells treated with TGF- $\beta 1$; the researchers concluded that the mechanism involved in alveolar EMT was probably Smad2-dependent [44]. Yu et al. observed that ginsenoside Rg1 attenuated TGF- $\beta 1$ induced EMT in HepG2 cells and might prevent invasion and migration [28]. Cho et al. reported that $\operatorname{Rg} 1$ inhibited TGF- $\beta 1$-induced myofibroblast differentiation in nasal polypderived fibroblasts [45]. In addition, it has been indicated that the extract of Panax ginseng inhibits TGF- $\beta 1$-mediated fibrosis by suppressing the phosphorylation of Smad 2 and Smad3 [32]. Likewise, we showed that ginsenoside Rg1 decreased the level of TGF- $\beta 1$ in serum and lung tissue of COPD rats induced by CS exposure. TGF- $\beta 1$-induced EMT is primarily dependent on Smad pathway initiated by TGF$\beta 1$ binding to its receptor and activation of the receptor complex. The present study showed that ginsenoside Rg1 dose-dependently decreased the protein expression of TGF$\beta 1 \mathrm{R}$, which inhibited the signal transduction of TGF- $\beta 1$, and consequently exerted an inhibitory effect on TGF- $\beta 1$-induced EMT. In addition, our data also showed that ginsenoside $\mathrm{Rg} 1$ attenuated the activation of Smad2 and Smad3 in vivo and in vitro. Therefore, we believe that the suppression of EMT by ginsenoside Rg1 was at least partly associated with the reduced production of TGF- $\beta 1$ and might be related to inhibition of the TGF- $\beta 1 /$ Smad signaling pathway. Interestingly, Mahmood et al. found the significant correlations between Smad2/3 and Smad7 expression with both an EMT activity marker and airflow obstruction [9]. Work on Smad7 is included in our future goals. To assess whether ginsenoside Rg1 attenuated EMT independent of the reduction of TGF$\beta 1$, we investigated the effects of ginsenoside Rg1 on HBE cells treated with TGF- $\beta 1$. The consistent outcome indicated that ginsenoside Rg1 treatment decreased the protein level of $\alpha$-SMA and alleviated the loss of E-cad in HBE cells compared with TGF- $\beta 1$ alone. Moreover, our in vitro study demonstrated that ginsenoside Rg1 mimicked the effect of SB525334, an inhibitor of TGF- $\beta 1$-induced Smad2/3 nuclear translocation, on reduction of $\alpha$-SMA and restoration of E-cad. Furthermore, SB525334 enhanced the suppression of EMT in the presence of ginsenoside Rg1, eliminating the involvement of TGF- $\beta 1 /$ Smad signaling in ginsenoside Rg1-mediated inhibition of EMT, which may provide novel therapeutic targets for COPD.

However, the mechanism of ginsenoside Rgl-evoked TGF- $\beta 1$ downregulation remains unclear. Current research indicated that ginsenoside Rg1 protected primary cultured cortical neurons from $\mathrm{A} \beta 25$-35-induced toxicity in a NF- $\kappa \mathrm{B}$ dependent manner [46]. NF- $\kappa \mathrm{B}$ activation could stimulate
TGF- $\beta 1$ production in vitro [47]. Therefore, NF- $\kappa \mathrm{B}$ is probably involved in ginsenoside Rgl-mediated decrease in TGF$\beta 1$ and the underlying mechanisms need to be further elucidated.

It should be noted that the current study has been implemented only on $\mathrm{HBE}$ cells in vivo and focused on classically described structure changes and proteins in EMT. Moreover, we could not fully determine the origin of fibroblasts/myofibroblasts accumulated in the surrounding bronchial epithelium in COPD. These could be locally produced or bone marrow-derived or even associated with migration and infiltration of mesenchymal cells in vessels around the bronchus. Indeed, we observed that $\alpha$-SMA expression was significantly increased in pulmonary vessels in COPD (Supplementary Figure S1 in Supplementary Material available online at https://doi.org/10.1155/2017/7171404). We will follow up with a detailed analysis of upstream pathways and EMT-related transcription factors in vivo and in vitro. Additionally, we will further explore the potential mechanism of ginsenoside Rgl using a cell culture approach including fibroblast cells and vascular endothelial cells.

In conclusion, the present study demonstrated that ginsenoside Rgl attenuated the progression of EMT, in both CS-induced COPD rat model and HBE cells exposed to CES, which was at least partly mediated by inhibition of the TGF- $\beta 1 /$ Smad pathway. The exact mechanisms underlying the beneficial effects of ginsenoside Rg1 warrant further investigation. Nevertheless, our current findings indicate that ginsenoside Rgl could act as an attractive therapeutic target for intervention against airway remodeling in COPD.

\section{Abbreviations \\ EMT: Epithelial-mesenchymal transition \\ COPD: Chronic obstructive pulmonary disease \\ CS: $\quad$ Cigarette smoke \\ CSE: Cigarette smoke extract \\ HBE: Human bronchial epithelial \\ TGF- $\beta 1$ : Transforming growth factor- $\beta 1$ \\ $\alpha$-SMA: $\alpha$-Smooth muscle actin \\ E-cad: Epithelial cadherin.}

\section{Conflicts of Interest}

The authors indicate no potential conflicts of interest regarding the publication of the paper.

\section{Authors' Contributions}

Xuejun Guo and Qian Liu were responsible for experimental design. Sibin Guan performed most of the experiments. Fengfeng Han and Wen Gu performed some of the cell experiments. Lin Song and Wenjing Ye analyzed the data. Sibin Guan and Weiguo $\mathrm{Xu}$ wrote the manuscript and prepared the figures. Xuejun Guo gave helpful suggestions on the manuscript. All authors read and approved the final manuscript. 


\section{Acknowledgments}

This work was supported by the National Natural Science Foundation of China (no. 81400026) and Youth Research Project of Shanghai Health and Family Planning Commission (no. 20134Y015). The funders had no role in research's design, data collection, or analysis, nor were they involved in the decision to publish or the preparation of the manuscript.

\section{References}

[1] R. Lozano, M. Naghavi, K. Foreman et al., "Global and regional mortality from 235 causes of death for 20 age groups in 1990 and 2010: A systematic analysis for the Global Burden of Disease Study 2010," The Lancet, vol. 380, no. 9859, pp. 2095-2128, 2012.

[2] M. Roth, "Airway and lung remodelling in chronic pulmonary obstructive disease: a role for muscarinic receptor antagonists?" Drugs, vol. 75, no. 1, pp. 1-8, 2015.

[3] S. G. Royce, Y. Moodley, and C. S. Samuel, "Novel therapeutic strategies for lung disorders associated with airway remodelling and fibrosis," Pharmacology \& Therapeutics, vol. 141, pp. 250260, 2014

[4] S. S. Sohal, C. Ward, W. Danial, R. Wood-Baker, and E. H. Walters, "Recent advances in understanding inflammation and remodeling in the airways in chronic obstructive pulmonary disease," Expert Review of Respiratory Medicine, vol. 7, no. 3, pp. 275-288, 2013.

[5] S. S. Sohal, D. Reid, A. Soltani et al., "Reticular basement membrane fragmentation and potential epithelial mesenchymal transition is exaggerated in the airways of smokers with chronic obstructive pulmonary disease," Respirology, vol. 15, no. 6, pp. 930-938, 2010.

[6] U. Valcourt, M. Kowanetz, H. Niimi, C.-H. Heldin, and A. Moustakas, "TGF- $\beta$ and the Smad signaling pathway support transcriptomic reprogramming during epithelial-mesenchymal cell transition," Molecular Biology of the Cell, vol. 16, no. 4, pp. 1987-2002, 2005.

[7] S. Hu, W. Yu, T.-J. Lv, C.-S. Chang, X. Li, and J. Jin, "Evidence of TGF- $\beta 1$ mediated epithelial-mesenchymal transition in immortalized benign prostatic hyperplasia cells," Molecular Membrane Biology, vol. 31, no. 2-3, pp. 103-110, 2014.

[8] L. Pang, Q. Li, C. Wei et al., "TGF- $\beta 1 /$ Smad signaling pathway regulates epithelial-to-mesenchymal transition in esophageal squamous cell carcinoma: in vitro and clinical analyses of cell lines and nomadic Kazakh patients from northwest Xinjiang, China," PLoS ONE, vol. 9, no. 12, Article ID el12300, 2014.

[9] M. Q. Mahmood, D. Reid, C. Ward et al., “Transforming growth factor (TGF) betal and Smad signalling pathways: A likely key to EMT-associated COPD pathogenesis," Respirology (Carlton, Vic.), vol. 22, pp. 133-140, 2017.

[10] H. Ikushima and K. Miyazono, "TGFB 2 signalling: a complex web in cancer progression," Nature Reviews Cancer, vol. 10, no. 6, pp. 415-424, 2010.

[11] S. Han, N. T. Bui, M. T. Ho, Y. M. Kim, M. Cho, and D. B. Shin, "Dexamethasone inhibits TGF- $\beta 1$-induced cell migration by regulating the ERK and AKT pathways in human colon cancer cells via CYR61," Cancer Research and Treatment, vol. 48, no. 3, pp. 1141-1153, 2016.

[12] J.-H. Park, J. Yoon, K. Y. Lee, and B. Park, "Effects of geniposide on hepatocytes undergoing epithelial-mesenchymal transition in hepatic fibrosis by targeting TGF $\beta /$ Smad and ERK-MAPK signaling pathways," Biochimie, vol. 113, article no. 4678, pp. 2634, 2015.

[13] B. Iskender, K. Izgi, and H. Canatan, "Novel anti-cancer agent myrtucommulone-A and thymoquinone abrogate epithelialmesenchymal transition in cancer cells mainly through the inhibition of PI3K/AKT signalling axis," Molecular and Cellular Biochemistry, vol. 416, no. 1-2, pp. 71-84, 2016.

[14] S.-L. Zhou, Z.-J. Zhou, Z.-Q. Hu et al., "CXCR2/CXCL5 axis contributes to epithelial-mesenchymal transition of HCC cells through activating PI3K/Akt/GSK-3 $\beta /$ Snail signaling," Cancer Letters, vol. 358, no. 2, pp. 124-135, 2015.

[15] X. Liu, Z. Li, Y. Song et al., "AURKA induces EMT by regulating histone modification through $\mathrm{Wnt} / \beta$-catenin and PI3K/Akt signaling pathway in gastric cancer," Oncotarget, vol. 7, no. 22, pp. 33152-33164, 2016.

[16] J. Wang, S. Li, Y. Fan et al., "Anti-fatigue activity of the watersoluble polysaccharides isolated from Panax ginseng C. A. Meyer," Journal of Ethnopharmacology, vol. 130, no. 2, pp. 421423, 2010.

[17] C. T. Nguyen, T. T. Luong, S. Y. Lee et al., "Panax ginseng aqueous extract prevents pneumococcal sepsis in vivo by potentiating cell survival and diminishing inflammation," Phytomedicine, vol. 22, no. 11, pp. 1055-1061, 2015.

[18] C. Z. Wang, S. Anderson, W. Du, T. C. He, and C. S. Yuan, "Red ginseng and cancer treatment," Chinese Journal of Natural Medicines, vol. 14, pp. 7-16, 2016.

[19] B.-Y. Hu, X.-J. Liu, R. Qiang et al., “Treatment with ginseng total saponins improves the neurorestoration of rat after traumatic brain injury," Journal of Ethnopharmacology, vol. 155, no. 2, pp. 1243-1255, 2014.

[20] S. Kang and H. Min, "Ginseng, the 'immunity boost': The effects of panax ginseng on immune system," Journal of Ginseng Research, vol. 36, no. 4, pp. 354-368, 2012.

[21] D. Gross, Z. Shenkman, B. Bleiberg, M. Dayan, M. Gittelson, and R. Efrat, "Ginseng improves pulmonary functions and exercise capacity in patients with COPD," Monaldi Archives for Chest Disease, vol. 57, pp. 242-246, 2002.

[22] D. Sun, B. Wang, M. Shi et al., "Pharmacokinetic, tissue distribution and excretion of ginsenoside-Rd in rodents," Phytomedicine, vol. 19, no. 3-4, pp. 369-373, 2012.

[23] L. Li, Y.-X. Sheng, J.-L. Zhang, S.-S. Wang, and D.-A. Guo, "High-performance liquid chromatograhic assay for the active saponins from Panax notoginseng in rat tissues," Biomedical Chromatography, vol. 20, no. 4, pp. 327-335, 2006.

[24] Q. F. Xu, X. L. Fang, and D. F. Chen, "Pharmacokinetics and bioavailability of ginsenoside Rb1 and Rg1 from Panax notoginseng in rats," Journal of Ethnopharmacology, vol. 84, no. 2-3, pp. 187-192, 2003.

[25] N. Li, Y. Liu, W. Li et al., "A uplc/ms-based metabolomics investigation of the protective effect of ginsenosides $\mathrm{rgl}$ and $\mathrm{rg} 2$ in mice with alzheimer's disease," Journal of Ginseng Research, vol. 40, no. 1, pp. 9-17, 2016.

[26] Y. Fan, J. Xia, D. Jia et al., "Mechanism of ginsenoside Rg1 renal protection in a mouse model of d-galactose-induced subacute damage," Pharmaceutical Biology, vol. 54, no. 9, pp. 1815-1821, 2016.

[27] S. Bao, Y. Zou, B. Wang et al., "Ginsenoside Rg1 improves lipopolysaccharide-induced acute lung injury by inhibiting inflammatory responses and modulating infiltration of M2 macrophages," International Immunopharmacology, vol. 28, no. 1, pp. 429-434, 2015. 
[28] M. Yu, X. Yu, D. Guo et al., "Ginsenoside Rg1 attenuates invasion and migration by inhibiting transforming growth factor- $\beta 1$ induced epithelial to mesenchymal transition in HepG2 cells," Molecular Medicine Reports, vol. 11, no. 4, pp. 3167-3173, 2015.

[29] X.-S. Xie, M. Yang, H.-C. Liu, C. Zuo, H.-J. Li, and J.-M. Fan, "Ginsenoside Rg1, a major active component isolated from Panax notoginseng, restrains tubular epithelial to myofibroblast transition in vitro," Journal of Ethnopharmacology, vol. 122, no. 1, pp. 35-41, 2009.

[30] A. Janoff and H. Carp, "Possible mechanisms of emphysema in smokers: cigarette smoke condensate suppresses protease inhibition in vitro," The American Review of Respiratory Disease, vol. 116, pp. 65-72, 1977.

[31] W. Gu, L. Song, X. M. Li, D. Wang, X. J. Guo, and W. $\mathrm{G}$. $\mathrm{Xu}$, "Mesenchymal stem cells alleviate airway inflammation and emphysema in COPD through down-regulation of cyclooxygenase-2 via p38 and ERK MAPK pathways," Scientific Reports, vol. 5, article 8733, 2015.

[32] J.-Y. Ahn, M.-H. Kim, M.-J. Lim et al., "The inhibitory effect of ginsan on TGF- $\beta$ mediated fibrotic process," Journal of Cellular Physiology, vol. 226, no. 5, pp. 1241-1247, 2011.

[33] E. Papakonstantinou, G. Karakiulakis, S. Batzios et al., "Acute exacerbations of COPD are associated with significant activation of matrix metalloproteinase 9 irrespectively of airway obstruction, emphysema and infection," Respiratory Research, vol. 16, no. 1, article 78, 2015.

[34] J. L. Shergis, Y. M. Di, A. L. Zhang et al., "Therapeutic potential of Panax ginseng and ginsenosides in the treatment of chronic obstructive pulmonary disease," Complementary Therapies in Medicine, vol. 22, no. 5, pp. 944-953, 2014.

[35] Y. L. Zhao, R. T. Zhu, and Y. L. Sun, "Epithelial-mesenchymal transition in liver fibrosis," Biomedical Reports, vol. 4, pp. 269274, 2016.

[36] J. Bennett, H. Cassidy, C. Slattery, M. P. Ryan, and T. McMorrow, "Tacrolimus modulates TGF-beta signaling to induce epithelial-mesenchymal transition in human renal proximal tubule epithelial cells," Journal of Clinical Medicine, vol. 5, 2016.

[37] N. Zidar, E. Boštjančič, M. Jerala et al., "Down-regulation of microRNAs of the miR-200 family and up-regulation of Snail and Slug in inflammatory bowel diseases - hallmark of epithelial-mesenchymal transition," Journal of Cellular and Molecular Medicine, vol. 20, no. 10, pp. 1813-1820, 2016.

[38] M. Q. Mahmood, S. S. Sohal, S. D. Shukla et al., "Epithelial mesenchymal transition in smokers: Large versus small airways and relation to airflow obstruction," International Journal of COPD, vol. 10, no. 1, pp. 1515-1524, 2015.

[39] T. Ijaz, K. Pazdrak, M. Kalita et al., "Systems biology approaches to understanding epithelial mesenchymal transition (EMT) in mucosal remodeling and signaling in asthma," World Allergy Organization Journal, vol. 7, no. 1, article no. 13, 2014.

[40] H. Sun, J. Chen, W. Qian et al., "Integrated long noncoding RNA analyses identify novel regulators of epithelialmesenchymal transition in the mouse model of pulmonary fibrosis," Journal of Cellular and Molecular Medicine, vol. 20, no. 7, pp. 1234-1246, 2016.

[41] L. Xie, D. Zhou, J. Xiong, J. You, Y. Zeng, and L. Peng, "Paraquat induce pulmonary epithelial-mesenchymal transition through transforming growth factor- $\beta 1$-dependent mechanism," Experimental and Toxicologic Pathology, vol. 68, no. 1, pp. 69-76, 2016.

[42] T.-L. Hackett, S. M. Warner, D. Stefanowicz et al., "Induction of epithelial-mesenchymal transition in primary airway epithelial cells from patients with asthma by transforming growth factor$\beta 1$," American Journal of Respiratory and Critical Care Medicine, vol. 180, no. 2, pp. 122-133, 2009.

[43] A. Gardner, A. J. Fisher, C. Richter et al., "The critical role of TAK1 in accentuated epithelial to mesenchymal transition in obliterative bronchiolitis after lung transplantation," American Journal of Pathology, vol. 180, no. 6, pp. 2293-2308, 2012.

[44] H. Kasai, J. T. Allen, R. M. Mason, T. Kamimura, and Z. Zhang, "TGF- $\beta 1$ induces human alveolar epithelial to mesenchymal cell transition (EMT)," Respiratory Research, vol. 6, article 56, 2005.

[45] J.-S. Cho, Y.-M. Moon, J.-Y. Um, J.-H. Moon, I.-H. Park, and H.M. Lee, "Inhibitory effect of ginsenoside Rg1 on extracellular matrix production via extracellular signal-regulated protein kinase/activator protein 1 pathway in nasal polyp-derived fibroblasts," Experimental Biology and Medicine, vol. 237, no. 6, pp. 663-669, 2012.

[46] J. Wu, H. Yang, Q. Zhao, X. Zhang, and Y. Lou, "Ginsenoside Rg1 exerts a protective effect against A2535induced toxicity in primary cultured rat cortical neurons through the NF-B/NO pathway," International Journal of Molecular Medicine, vol. 37, no. 3, pp. 781-788, 2016.

[47] P. Rameshwar, R. Narayanan, J. Qian, T. N. Denny, C. Colon, and P. Gascon, "NF-kappa B as a central mediator in the induction of TGF-beta in monocytes from patients with idiopathic myelofibrosis: an inflammatory response beyond the realm of homeostasis," Journal of Immunology, vol. 165, pp. 2271-2277, 2000. 


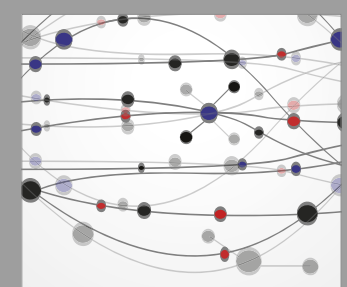

The Scientific World Journal
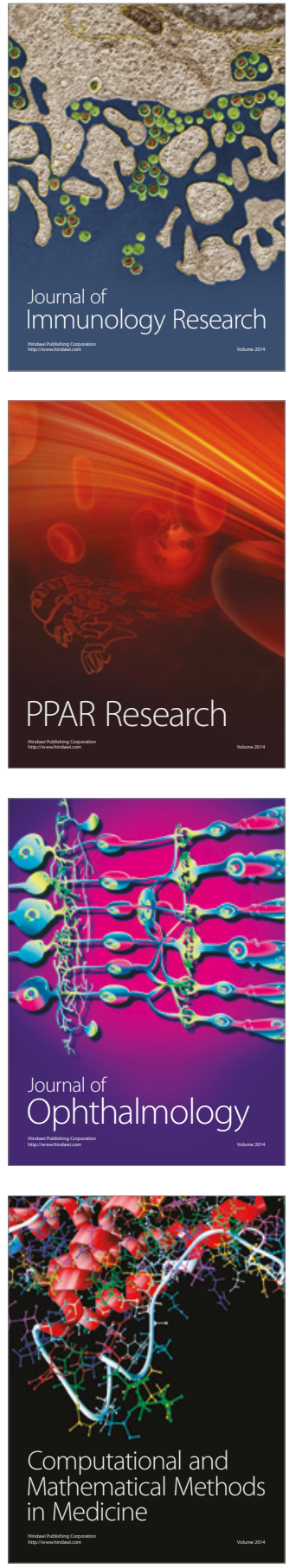

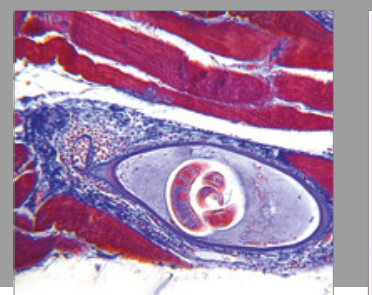

Gastroenterology Research and Practice
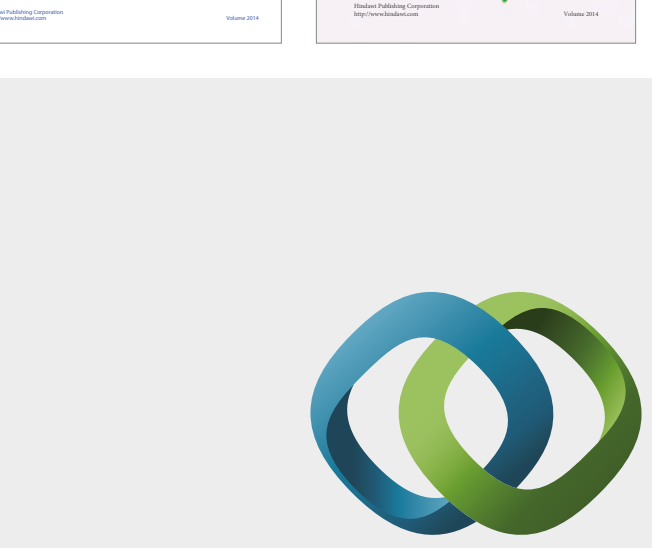

\section{Hindawi}

Submit your manuscripts at

https://www.hindawi.com
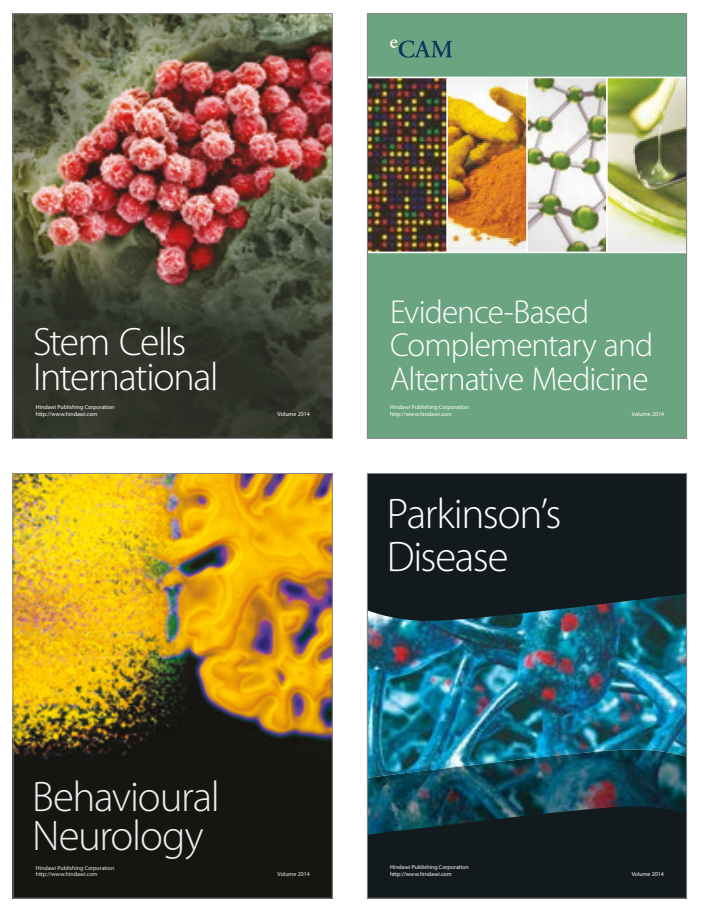
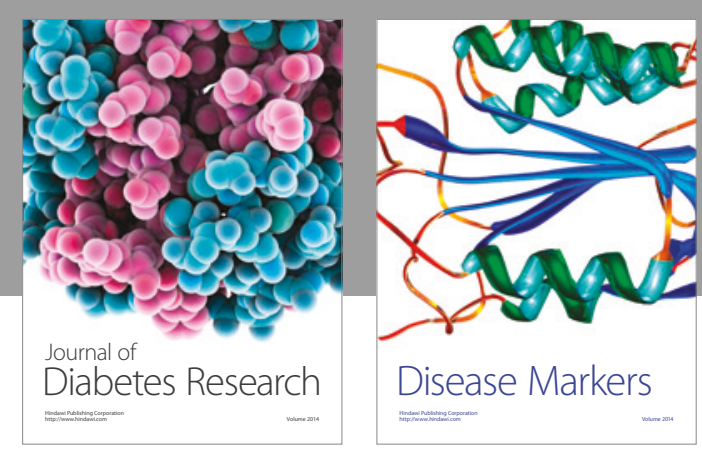

Disease Markers
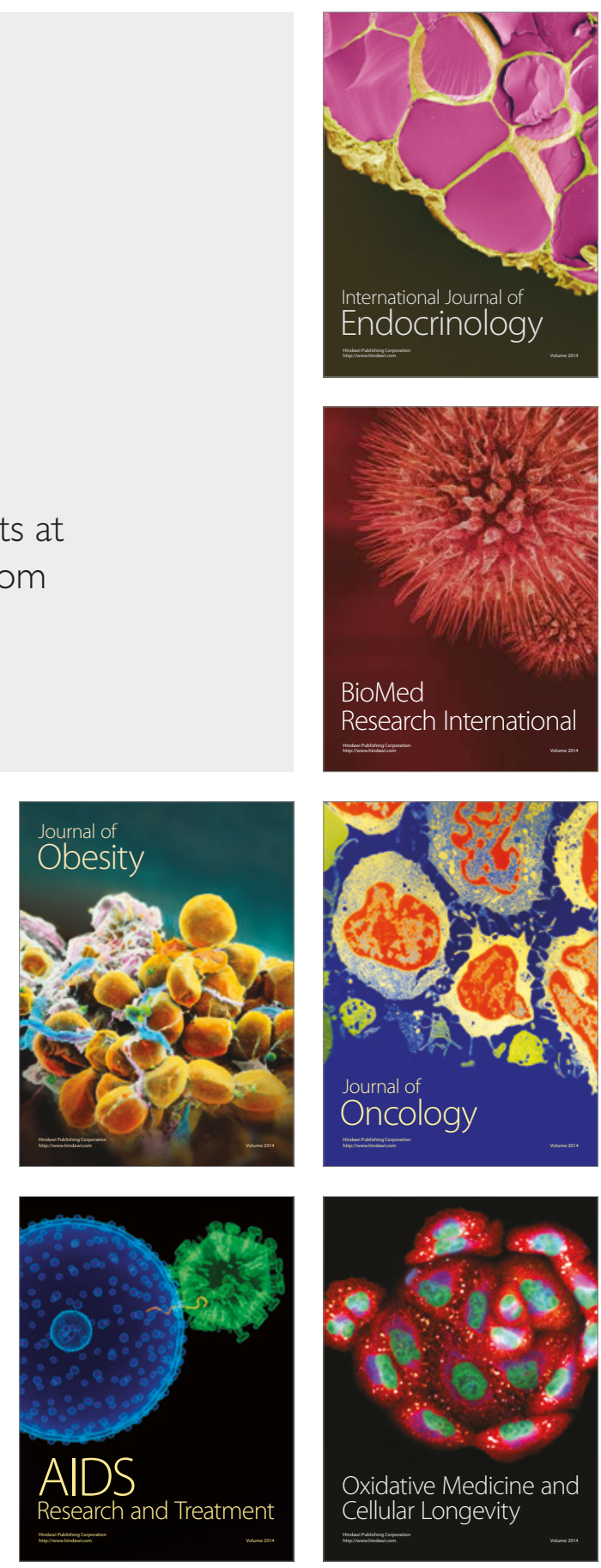